\title{
Design and development of composite anodic bonding system based on dielectric barrier discharge method
}

\author{
Xufeng Zhao \\ Robotics and Microsystems Center \& \\ Innovation Center of Suzhou Nano Science and \\ Technology, \\ Soochow University \\ Suzhou 215123, China \\ ZhaoXuFenZ@163.com \\ Mingqiang Pan \\ Robotics and Microsystems Center \& \\ Innovation Center of Suzhou Nano Science and \\ Technology, \\ Soochow University, \\ Suzhou 215123, China \\ pmq@suda.edu.cn
}

\author{
Chunyi Lu \\ Robotics and Microsystems Center \& \\ Innovation Center of Suzhou Nano Science and \\ Technology, \\ Soochow University, \\ Suzhou 215123, China \\ clu.ai.fan@163.com
}

\section{Liguo Chen}

Robotics and Microsystems Center \& Innovation Center of Suzhou Nano Science and

Technology,

Soochow University,

Suzhou 215123, China

chenliguo@suda.edu.cn

\begin{abstract}
In this paper, a composite anodic bonding system is developed. In order to reduce the temperature of anodic bonding, DBD(Dielectric barrier discharge)plasma treatment is used. The process of composite anodic bonding is described. The effects and mechanism of the DBD plasma on bond quality are investigated. The functional of composite anodic bonding system is determined. The composite bonding system can be modular constructed by the power supply module, temperature module, motion operation module, visual detection module. When the temperature is $300^{\circ} \mathrm{C}$, the DBD Voltage is $1200 \mathrm{v}$, frequency is $20 \mathrm{KHz}$, interspace is $2 \mathrm{~mm}$, glow-like plasmas is generated at atmospheric pressure, and bonding quality is best. DBD treatment can reduce the bonding temperature is proved through experiment.
\end{abstract}

Keywords-DBD;anodic bonding; plasma;surface treatment;gas discharge

\section{INTRODUCTION}

Anodic bonding is one of the key process for the construction and packaging of micro-electro-mechanical systems(MEMs)[1].This technique has been used in fabrication process of many different microdevices, such as pressure sensors and micropumps. Anodic bonding of silicon to glass allows for 3Dstructure fabrication used in microfluidic devices[2]. The process of anodic bonding is simple, and the bonding strength is high enough in many cases. Therefore anodic bonding is widely used in vacuum packaging, hermetic sealing and encapsulation.
In anodic bonding, the substrates are heated to a typical temperature of $400-450^{\circ} \mathrm{C}$, and a voltage of 400 $1000 \mathrm{v}$ is applied to silicon and glass. Finally, electrostatic force makes the alkali metal ion in glass move to the side of positive electrode. The migration of ions leads to an irreversible chemical bond at the boundary layer between the glass and silicon. A special type of glass named Pyrex7740 is the most commonly used in anodic bonding because of its large sodium cation content and the matched thermal expansion coefficient with that of silicon[3].

Sometimes, high temperature will damage prefabricated devices and integrated circuitry. The wafers used in anodic bonding may have large difference in thermal expansion coefficient, and it will cause bondinginduced stress problem and warpage after cooling. So, it is necessary to reduce the temperature in the anodic bonding process. Currently, the mainstream of wafer bonding focuses on achieving strong wafer bonding at temperature as low as possible. However,the bonding strength decrease with the decrease of temperature in anodic bonding process. Low bonding strength will bring in the bubbles or cavities at the interface. More and more interests of researchers are focused on low-temperature bonding (the maximum process temperature below $300 \circ \mathrm{C}$ )[4].

M.M.R. Howlader[5] achieved anodic bonding between germanium and silicon at $200^{\circ} \mathrm{C}$ with plasma surface treatment. J wei[6] achieve anodic boning between silicon and glass at $200{ }^{\circ} \mathrm{C}$ to $300{ }^{\circ} \mathrm{C}$ with chemical surfactants. The bonding strength is over $10 \mathrm{Mpa}$. 
Non equilibrium atmospheric-pressure plasmas are widely used in surface treatment and biomedical applications, as they have recently been made possible for plasma direct treatment without expensive and complicated vacuum system[7]. In the past few years, many atmospheric pressure plasmas have been developed, including dielectric-barrier discharge (DBD) [8-12].

In this work, a novel method of anodic bonding in order to reduce the temperature during anodic bonding is studied, and the anodic bonding system is developed.

\section{MECHANISM OF DBD}

\section{A. The introduction of dielectric barrier discharge}

Dielectric barrier discharge(DBD) is also known as barrier discharge(BD) or silent discharge (SD).DBD is a gas discharge with dielectric inserted into the discharge space. The dielectric material can be covered on the electrode or suspended in the discharge space. When a sufficiently high AC voltage is applied between the two electrodes, discharge will generate between the electrodes. Because of the dielectric material, the forming of spark discharge or arc discharge in discharge gap is avoid. what's more, dielectric can stabilize the discharge uniformly distributed in the entire discharge space.

The interaction of dielectric barrier discharge plasma with the silicon / glass surface consists of two main stages: the physicochemical processes of active particles generating in air; the interaction of active particles generated in air DBD with silicon / glass surfaces.

\section{B. The physical processes of air DBD}

In the air DBD,the value of pressure and discharge gap is large. The initial process of discharging mechanism can explain by the streamer discharge. In the streamer discharge phase, the discharge space is filled with a random distribution of transient stream. Then,the stream region expands to anode rapidly. Eventually, a filamentary discharge channel with high conductivity through the discharge space is produced. With the dielectric introduced to discharge space, the free rise of the discharge current is restrict.,therefore the formation of a spark or arc discharge between the electrodes is prevented. A plurality of electron is produced in discharge process. When these electrons reached the anode, these electrons will gather down on the surface of the dielectric. The electrons gathered on the surface of dielectric will produce an additional electric field in the opposite direction of the electric field. With the increase of the charge accumulation on the media, the additional electric field is also enhanced. The total electric field strength in the gap will drop with the enhancing of additional electric field. When the total electric field is less then the breakdown strength, the discharge will stop. So it is needed to use AC power supply.

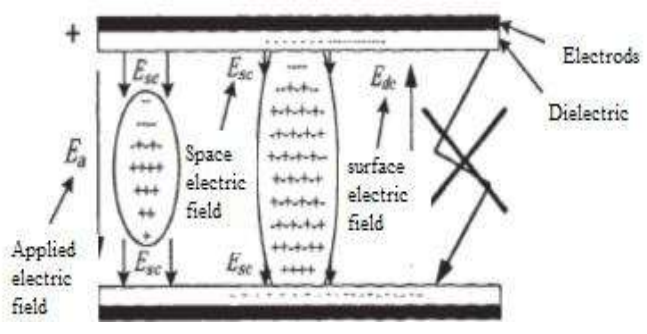

Figure 1. DBD process.

\section{The affect of the plasma}

Through the DBD plasma treatment, the interface of glass are terminated by hydroxyl groups. Hydrogen bond energy is about $0.2-0.3 \mathrm{eV}$, as shown in Fig.3. However, the electronic produced in DBD process is about $4-5 \mathrm{eV}$. So, the electronic have enough energy to break the hydrogen bond. When the hydrogen bond is broken by electronic in the plasma, the surface of the glass will be terminated by hydroxyl groups. The process is showed in Fig. 2.

When the silicon contact to the glass which is DBD plasma treated, chemical reactions occur as follows.

\section{$\mathrm{Si}-\mathrm{OH}+\mathrm{HO}-\mathrm{Si} \leftrightarrow \mathrm{Si}-\mathrm{O}-\mathrm{Si}+\mathrm{HOH}$}

Due to the glass surface are terminated by hydroxyl groups, hydrogen bond is produced when the silicon contact to glass. Hydroxyl bonds will narrow the distance between the surface of silicon and glass. Closer contact between silicon and glass cause higher electric force during anodic bonding. That is why the DBD process imposes a positive effect on bond quality improvement

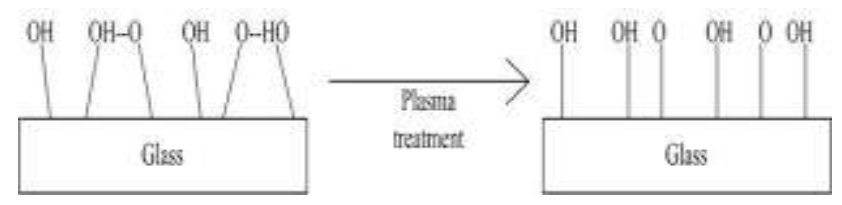

Figure 2. Plasma treatment process.

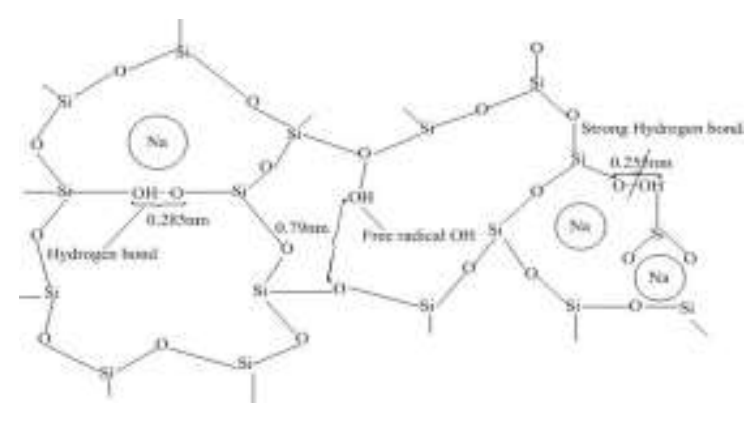

Figure 3. Glass structure[13].

\section{DEVELOPMENT OF COMPOSITE ANODIC BONDING} SYSTEM

From the analysis of composite anodic bonding process, the requirements are as following :

1.For silicon wafers and glass dielectric barrier discharge process conditions: voltage AC500-2000V, discharge frequency $10-10000 \mathrm{~Hz}$, discharge gap 0.1 $10 \mathrm{~mm}$; therefore function of system is required as following: 
1)High voltage AC power supply is needed. Voltage and frequency of the AV power supply should be adjustable.

2)Precision Z-axis is needed for adjusting the discharge gap.

3)Interface leveling decoupling mechanism. It is used to adjust the discharge gap of the two parallel planes.

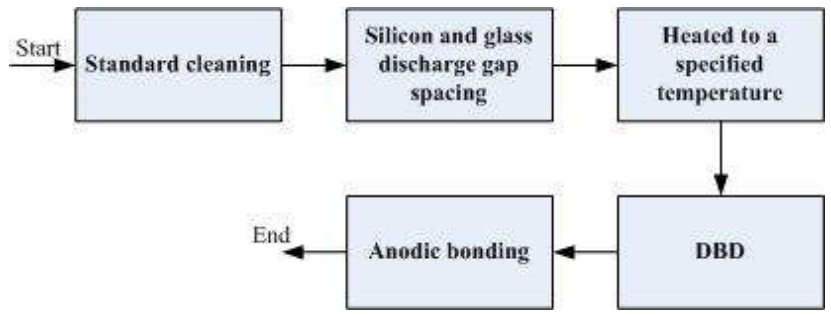

Figure 4. Composite anodic bonding process

2.Anodic bonding process conditions: heating temperature of $200{ }^{\circ} \mathrm{C}-450{ }^{\circ} \mathrm{C}$, pressure force 100-200gf, voltage 1000-1200VDC; therefore functional requirements:

1)High-voltage DC power supply, the power supply voltage or current is adjustable.

2)Robot requires three-dimensional movement for chip alignment and handling.

3)Bonding pressure control.

4)Bonding temperature control.

3.The timing of the two processes complex functional requirements:

1)Two kinds of high-voltage power switching and interference protection.

2)Design of a composite structure of mechanical movement.

3)Microscopic vision alignment.

4)Other auxiliary functions.

Based on the above analysis of the demand function, composite bonding system can be modular construction of the power supply module, temperature module, motion operation module, visual detection module.

\section{A. Design and implementation of motion modules}

Considering the dielectric barrier discharge and anodic bonding process,motion module needs to implement the wafer pickup, transportation, laying, posture adjustment, bonding pressure applying, discharge gap adjustment.Therefore, the motion module can be designed as shown in Figure 5. The module is consisted by XYZ coordinate precision linear motion platform, precision rotary motion platform.

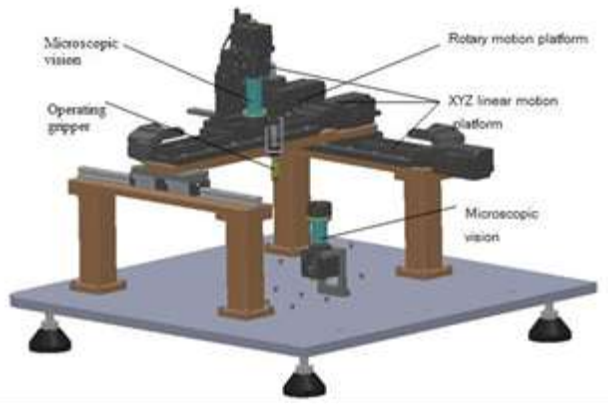

Figure 5. Movement operations module structure diagram

\section{B. Design and implementation of the power module}

Anodic bonding process requires a DC high voltage power supply, which is characterized by high voltage, current, voltage controllable. The dielectric barrier discharge plasma processes require high voltage variable frequency $\mathrm{AC}$ power, which is characterized by high voltage, voltage and frequency controllable. Considering the characteristics and timing of the two processes,complex high-voltage power is designed using an inverter boost.

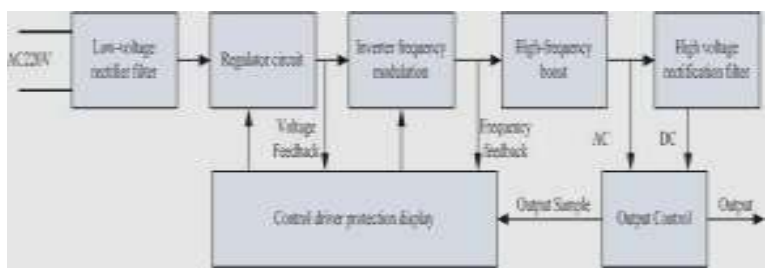

Figure 6. High voltage power supply diagram

\section{Design and implementation of operating gripper.}

In order to facilitate the combination, easy to replace, the operating gripper is modular designed. It is consisted by force sensing module and operating tool module[14]. The force sensing module is consisted by arm, the micro force sensor, the insulating member.Operating tool module is a vacuum suction device.

Vacuum system is the key for operating gripper to achieve chip pickup, release, handling. The Vacuum system is consisted by pressure source, pressure regulating valve, vacuum generator, electromagnetic switch valves and pressure sensors.

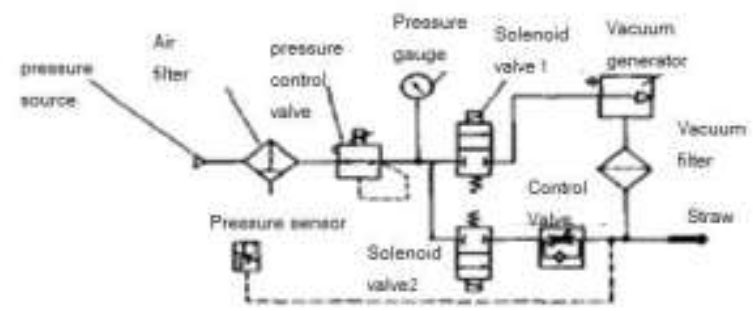

Figure 7. Vacuum system schematic

\section{EXPERIMENTAL STUDY OF THE ANODIC BONDING SYSTEM.}

In order to verify the effectiveness of the system,a complex anodic bonding experiment is conducted.The components of glass used in anodic bonding glass is very different with commonly glass. Borosilicate glass, PSG, Pyrex7740 and Pyrex7070 are commonly used. In this paper, pyrex 7740 glass is used.

TABLE I. PYREX7740 INGREDIENTS

\begin{tabular}{|l|c|c|c|c|c|}
\hline $\begin{array}{c}\text { Ingred } \\
\text { ient }\end{array}$ & $\mathrm{SiO}_{2}$ & $\mathrm{~B}_{2} \boldsymbol{O}_{3}$ & $\mathrm{Na}_{2} \mathrm{O}$ & $\mathrm{Al}_{\mathbf{2}} \mathrm{O}_{3}$ & $\mathrm{~K}_{\mathbf{2}} \mathrm{O}$ \\
\hline $\begin{array}{l}\text { Conten } \\
\text { t }\end{array}$ & 80.5 & 12.9 & 3.8 & 2.2 & 0.4 \\
\hline
\end{tabular}

Silicon wafer used in experimental is $3 \mathrm{~mm} \times 3 \mathrm{~mm} \times$ $0.5 \mathrm{~mm}$, and glass is $5 \mathrm{~mm} \times 5 \mathrm{~mm} \times 0.5 \mathrm{~mm}$.

Experimental procedure: 
1) Cleaning the silicon and glass wafer, then set the stack of silicon and glass on the down electrode. The glass is under the silicon and contact to the down electrode.

2) Control the operating gripper to stuck the silicon, then elevate the silicon by motion platform. The silicon and the glass is separated by a gap. The thickness of the gap is typically $1-2 \mathrm{~mm}$.

3) Apply appropriate voltage, then the DBD will occur between the silicon and glass. The DBD plasma modify the surface of silicon and glass.

4) Lower the silicon to make it contact to the glass. Apply a appropriate DC voltage to start the anodic bonding process.

When the temperature is $300^{\circ} \mathrm{C}$, the DBD Voltage is $1200 \mathrm{v}$, frequency is $20 \mathrm{KHz}$, interspace is $2 \mathrm{~mm}$, glowlike plasmas is generated at atmospheric pressure.By comparing the experimental group and control group results, it can be determined that DBD treatment can improve the bond quality. When the bonding conditions are the same, the bonded area of the group which is DBD treated is larger then the bonded area of control group.

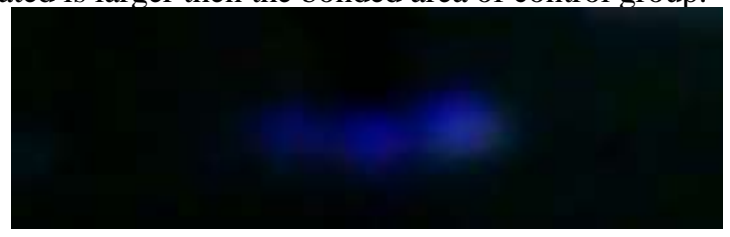

Figure 8. dielectric barrier discharge

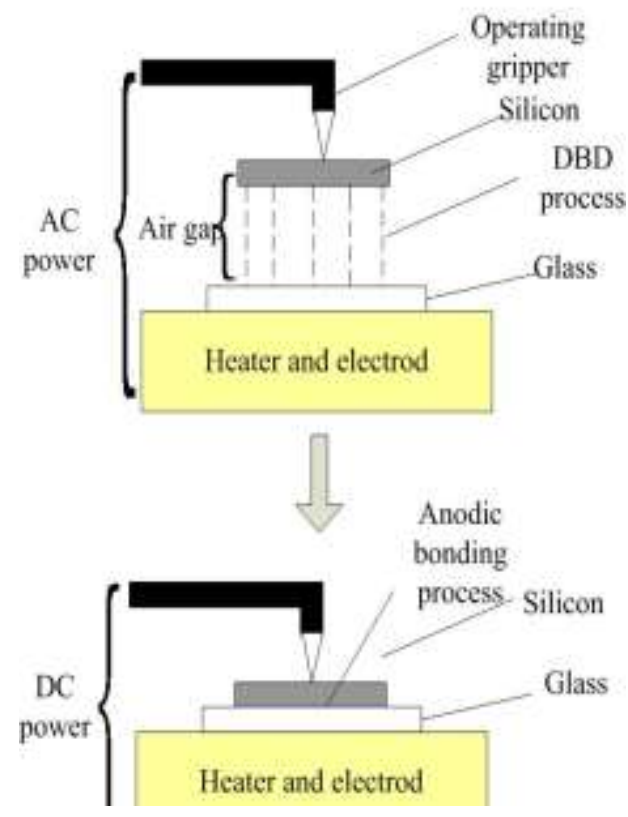

Figure 9. Discharge and anodic bonding conversion

Experimental results show that the impact of discharge voltage and gap on bonding quality is significant. When the DBD Voltage is 1200 , frequency is $20 \mathrm{KHz}$, interspace is $2 \mathrm{~mm}$, the bonding quality is best.

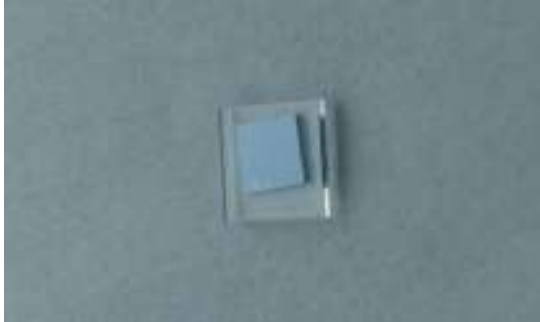

Figure 10. Bonded samples

\section{CONCLUSIONS}

In this paper, the composite anode bonding process based on DBD treatment is analyzed. The functional of composite anodic bonding system is determined. On the basis of the system functional description, the detail of the development of the operating gripper,motion modules, and other key technologies of the module are described. DBD treatment can improve the bond quality is proved. When the DBD Voltage is 1200 , frequency is $20 \mathrm{KHz}$, interspace is $2 \mathrm{~mm}$, the bonding quality is best.

\section{ACKNOWLEDGMENT}

The authors gratefully acknowledge the contribution of the National Natural Science Foundation of China (No. 61106110), the National High Technology Research and Development Program of China (No. 2011 AA040403 and No.2012AA040404).

\section{REFERENCES}

[1] G.Y. Li, L. Wang. Influence of bonding parameters on electrostatic force in anodic wafer bonding[J]. Thin Solid Film,2004,463:334-338.

[2] Hongbin $\mathrm{Yu}$, Guangya Zhou, Fook Siong Chau. Yield improvement for anodic bonding with suspending structure[J]. Sensors and Actuators A, 2008 ,143:462-468.

[3] JWei, H Xie, M L Nai, C KWong, et al. Low temperature wafer anodic bondin[J]. Micromech. Microeng, 2003, 13: 217-222.

[4] Chuai Rongyan, Liu Xiaowei , Chen Weiping, et al. Investigation on silicon-glass electrostatic bonding time[J]. Sensors and Actuators A, 2006, 127:194-199.

[5] M.M.R. Howlader. Hybrid plasma bonding of germanium and glass wafers at low temperature[J].Materials Letters, 2010 , 64(13):1532-1535.

[6] Mingxiang Chen, Author Vitae, Liulin Yuan Author Vitae, Sheng Liu. Research on low-temperature anodic bonding using induction heating[J]. Sensors and Actuators A: Physical, 2007, 133(1):266269.

[7] Jie Tang, Shibo Li,Wei Zhao. Development of a stable dielectricbarrier discharge enhanced laminar plasma jet generated at atmospheric pressure[J], APPLIED PHYSICS LETTERS, 2012,100:253505

[8] Thomas C C, Enloe C L, Wilkinson S P. Dielectric barrier discharge plasma actuators for flow control[J]. Annual Review of Fluid Mechanics, 2010, 42:505-529.

[9] Takashima K, Zuzeek Y, Lempert W R, et a1. Characterization of surface dielectric barrier discharge plasma sustained by repetitive nanose - cond pulses[J]. AIAA Paper, 2010-4764.

[10] Song Huimin,Jia Min,Liang Hua, et a1. Experimental investigation of the plasma aerodynamic actuation generated by nanosecondpulse sliding discharge $[\mathrm{C}] / /$ The Third International Conference on Measuring Technology and Mechattonics Automation: [s. n.], 2011 116-119. 
[11] Jiang Hui,Shao Tap,Yu Yang, et a1. Comparison of the characteristics in uanosecond-pulsed dielectric barrier discharge using different dielectric materials[J]. High Voltage Engineering , 2011, .37(6) : 1529-1535.

[12] Liu Yong, Li Qingquan.Study on properties of electrohydrodynamic(EHD)plasma actuator[J]. Advanced Technology of Electrical Engineering and Energy, 2009, 28(2):2932

[13] T Yamamoto, M Okubo, N Imai, Y Mori. Improvement on hydrophilic and hydrophobic properties of glass surface treated by nonthermal plasma induced by silent corona discharge[J]. Plasma Chemistry and Plasma Processing, 2004, 24(1):1-12.

[14] CHEN Li-guo, LING Yun-feng, CHEN Tao. Development of Automatic Microassembly System for MEMS Pressure Sensor Packaging[J]. Nanotechnology and Precision Engineering, 2008, 6(4):297-301. 